\title{
Convex Curvature Effect on the Wake Including Recovery from the Curvature
}

\author{
S. M. YOU \\ The University of Texas at Arlington, Department of Mechanical Engineering, Heat Transfer Laboratory, \\ Arlington, Texas 76019-0023
}

\begin{abstract}
Streamwise convex-curvature effect including recovery process is discussed. Previous studies have demonstrated that even mild streamwise curvature has a marked effect on turbulence structure, heat transfer and skin friction coefficient. This effect is further investigated in the present study with Coles wake law. Coles previously reported that flows in or near equilibrium have a constant wake parameter depending on the strength of streamwise pressure gradient. Turbulent boundary layers on the curved and following recovery wall may not be in or near equilibrium and the wake parameter may depend upon the strength of curvature, $\delta / R$. Different streamwise curvature strength, ranging $0.01-0.1$, is tested to confirm the validity of the wake law. The ratio of wake parameter between the curved and flat wall is found to be proportional to that of skin friction coefficient for the flows in both convexly curved and recovery sections. This relationship describes the deviation of wake behavior from the flat-plate turbulent boundary layer due to convex curvature.
\end{abstract}

Key Words: Convex surface; Streamwise curvature; Recovery; Wake; Turbulent; Boundary layer

A better understanding of transport process for flows over streamwise curved surfaces is required for many practical applications. Gas turbine is a representative application where accurate prediction of heat transfer and flow field is critical to a successful design. Because curvature and its recovery have significant influences on high-performance turbomachinery design, models must appropriately include these effects for the accurate predictions of momentum and heat transfer of the complex curved flow.

Turbine blade has two types of surfaces; the pressure side (concave) and suction side (convex). Previous studies indicated significant differences in heat transfer performance between the concave and convex surfaces, regarding heat transfer coefficient, skin friction and turbulence structures. As demonstrated by the Stanton number values in Thomann [1968], concave-side and convex-side heat transfer appeared to differ as much as $40 \%$ at the later section of the test wall. Concave surface showed an enhancement of heat transfer whereas convex surface indicated a decrease in heat transfer. In a comprehensive survey on the effects of streamline curvature, Bradshaw [1972] also pointed out that curvature effects on shear stress and heat transfer are significant even for cases of radii of curvature of more than a hundred times the shear layer thickness $(\delta / R<0.01)$, namely, weak curvature.

According to the work by Bradshaw [1972], boundary layer development with sustained weak curvature, $\delta / R=$ 0.01 , can be predicted with the curvature correction proposed as

$$
l / l_{o}=1+\beta \cdot R i \quad \text { where } \quad R i=2 \frac{U / R}{\partial U / \partial y}
$$

$l_{o}$ is the mixing length of the flat wall and $\beta$ is a constant. This model fails when the strength of curvature becomes strong. Meanwhile, in 1981, Adams and Johnston proposed a computational model for the curved turbulent boundary layers. Successful predictions were made for the strong-curved cases while their mixing-length model indicated some limitations in weak-curved cases. Adams and Johnston also pointed out that the discrepancy between the measured and predicted recovery region $C_{f}$ values is puzzling and deserves more study. The recovery process was initially experimented in strong-curved cases by Gillis et al. [1981, 1983] and Simon et al. 
[1982a, 1982b]. The test wall consisted of a developing flat section, a uniformly curved section, and a flat recovery section (Fig. 1). The disagreement of prediction in the recovery is clearly shown in Figure 2 for the experimental data $(\delta / R=0.05)$ of Gillis et al. [1981].

The eddy viscosity model with curvature modification from $k-\epsilon$ turbulence transport equations was used by Launder et al. [1977] and Rodi and Scheuerer [1983]. The effects of curvature are accounted for by making one of the coefficients in the transport equation for the energy dissipation as a function of the turbulent Richardson number. However, the slow response of the recovery section was not appropriately predicted.

An effort is, therefore, made to characterize the convex-curved turbulent boundary layer and its recovery. In the present study, it is tried to correlate the wake region with the viscous sublayer of convex-curved flows. A relationship is sought which can be extended to the slow recovery processes downstream of curvature. A successful correlation may result in a better model for the prediction of gas turbine blade flows.

\section{Previous Work}

As stated in a comprehensive survey on curvature effect by Bradshaw [1972], the history of the curvature effect study dates back to the early 1930s: Wilcken [1930], Wendt [1933], and Schmidbauer [1936]. In 1968, Thomann measured heat transfer coefficients on straight, convex, and concave surfaces at a free-stream Mach number of 2.5 . They showed that a concave wall increased the convective heat transfer by $20 \%$, and that a convex wall decreased it by the same amount, with $\delta / R<$

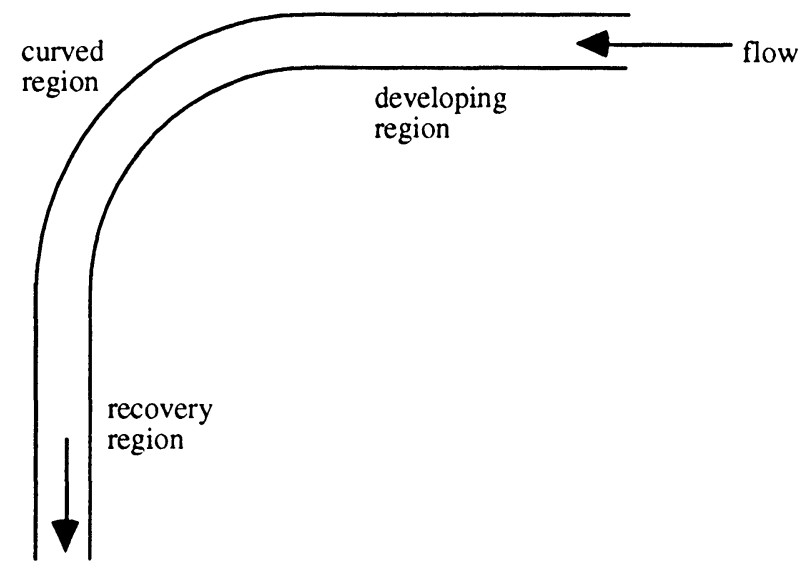

FIGURE 1 Curved boundary layer test facility; developing, curved and recovery regions.

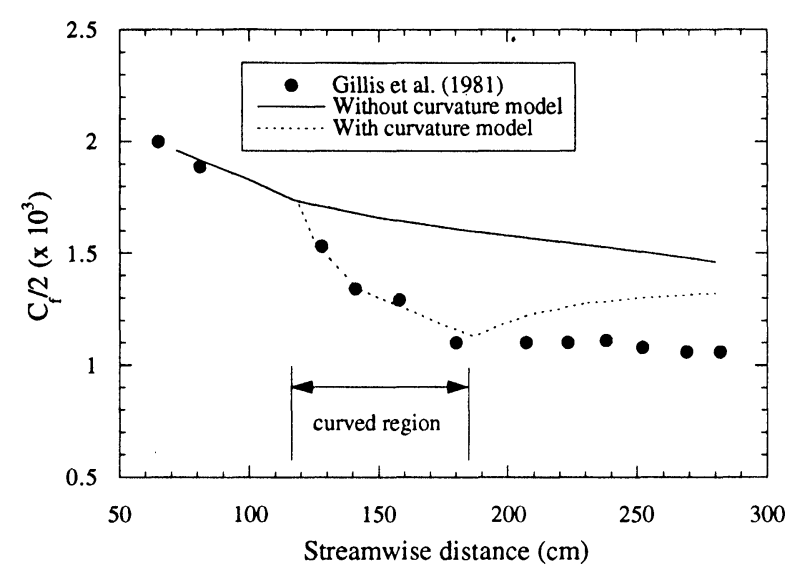

FIGURE 2 Prediction of skin friction coefficient for the third Gillis experiment $(\delta / R=0.05)$ by Adams and Johnston (1981).

0.02. Bradshaw [1969] suggested an analogy between streamline curvature and buoyancy in 1969 . He then proposed a curved-flow form of the Monin-Oboukhov formula, $l / l_{o}=l+\beta \cdot R i$ that showed great success in the prediction of curvature effects.

So and Mellor [1973] performed a detailed hydrodynamic experiment on curved walls while eliminating secondary flows. The aspect ratio was at least 6.2 throughout the test section to maintain two-dimensional flow. In the report, normal and shear stress profiles appeared to be self-similar after $30 \mathrm{~cm}$ downstream of entry to a strongly curved section $(\delta / R=0.078)$. Ellis and Joubert [1974] measured mean velocity profiles in two curved ducts of different radii, and they found that the law of the wall took a modified form; the width of the logarithmic zone was curvature dependent. Hoffmann and Bradshaw [1978] presented rather complete measurements with $\delta / R=0.01$. Their data included second, third and fourth-order mean products of velocity fluctuations and showed that curvature influences higher-order terms more than lower-order terms. Following Hoffmann and Bradshaw [1978], and using the same facility, Muck [1982] investigated curvature effects on boundary layers developing from a flat surface onto a mildly curved convex and concave surface of constant radius of curvature $(\delta / R=0.01)$. He observed that a mildly curved flow is qualitatively different from a strongly curved flow, and that the mechanisms of stabilizing (convex) curvature and destabilizing (concave) curvature are qualitatively different. The dominant structure of a spanwise array of steady Taylor-Görtler vortices existed for the concave surface (Schlichting [1951]).

Carefully controlled experiments on strongly convexcurved boundary layers were accomplished by Simon $e t$ 
al. [1982a, 1982b] and Gillis et al. [1981, 1983]. These experiments provided the beginning of a data base for recovery from curvature effects by introducing a flatrecovery wall after the flat-developing and curved walls-see Fig. 1. When the flow is strongly curved $(\delta / R>0.05)$, the turbulent transport mechanisms changed dramatically. Shear stress profiles measured by Gillis et al. [1983] within the curved region for the strong curvatures $(\delta / R=0.05$ and 0.1$)$, collapsed upon one another when plotted in $-u^{\prime} v^{\prime} / U_{\tau}^{2}$ vs. $y / R$ coordinates. The Stanton number response to the curvature was also nearly identical for the strong curvatures indicating a very weak dependence on $\delta / R$. Following the strongly curved cases, a parametric study of weak-to-intermediate strength $(0.01<\delta / R<0.05)$ curvature was experimentally investigated by You et al. [1986]. Varying the strength of curvature by changing only radius, $R$, they showed the asymptotic behavior suggested by Gillis and Johnston for flows with $\delta / R>0.04$. In You et al. [1986], recovery data was also measured for the weak-to-intermediate curvatures $(0.01-0.05)$. The effects of curvature and recovery on the turbulent transport quantities were reported by Kim and Simon [1987] using mild strength of curvature $(\delta / R=0.03)$.

\section{RESULTS AND DISCUSSION}

The experimental data of Simon et al. [1982b] and You et al. [1986] are chosen herein to investigate the mechanisms of the fast reduction and extremely slow recovery of skin friction at the curved and recovery sections, respectively. In order to systematically investigate the streamwise curvature effect, experimental data sets used in the present study consistently consist of a developing flat pre-plate, a uniform radius-of-curvature curved wall followed by a flat-plate recovery wall (Fig. 1). Descriptions of two strong curvature cases, TWS- 0.1 and TWS0.05 by Simon et al. [1982b], and two weak-to-moderate curvature cases, SMY-0.03 and SMY-0.01 by You et al.
[1986], are listed in Table I. The numbers, 0.1, 0.05, 0.03 and 0.01 , indicate the representative strength of curvature, $\delta / R$, for each case.

As seen in Fig. 3 for the four cases, the measured values of skin friction coefficient in the developing section are compared to a flat plate correlation by Ludwieg and Tillmann [1949] and these are in a good agreement. Ludwieg and Tillmann proposed, in 1949, the semi-empirical law describing skin friction coefficient variation on the flat-plate turbulent boundary layers;

$$
C_{f}=0.246 \cdot 10^{-0.678 H} R_{\theta}{ }^{-0.268}
$$

The effects of curvature on heat transfer and fluid mechanics are significant. According to the experiment of SMY-0.03 (You et al. [1986]), Stanton number responds immediately at the entry to the curve, decreasing about $15 \%$ by the streamwise distance $x=30 \mathrm{~cm}$ and continuing to decrease slowly thereafter. The same trend can be observed for the skin friction coefficient as shown in Fig. 4. Recovery of skin friction coefficient is even slower than that of the Stanton number. Stanton number increases to within $13 \%$ of the flat-plate value, but skin friction coefficient is still $20 \%$ below the flat-plate value. Although not shown here, $C_{f} / 2$ values for the weak and strong curvature cases in Table I were reduced significantly at the entry to the curve and the recovery process was slow. Though the four cases in Table I consistently showed the same effects of curvature on shear stress at the wall, the effects of the different strengths of curvature are not predictable.

\section{Law of the Wake for Flat-Plate Turbulent Boundary Layers: Theory}

The velocity profile of a turbulent boundary layer can be described by two matching layers-the inner layer and the outer layer. The law for the inner layer starts from an assumption that the mean velocity is a function of a dimensionless distance $Y^{+}$:

TABLE I

Description of the experiments

\begin{tabular}{|c|c|c|c|c|}
\hline Cases & TWS-0.1 & TWS-0.05 & SMY-0.03 & SMY-0.01 \\
\hline$R(\mathrm{~cm})$ & 45 & 45 & 90 & 210 \\
\hline$U_{p w}(\mathrm{~m} / \mathrm{sec})$ & 14.5 & 14.4 & 16.3 & 17.1 \\
\hline $\operatorname{Re}_{\theta} *$ & 3613 & 1888 & 2737 & 2916 \\
\hline$\delta / R$ & $0.071-0.113$ & $0.039-0.078$ & $0.024-.054$ & $0.010-0.024$ \\
\hline Developing section & stations $5-6$ & station 5 & station 1 & station 1 \\
\hline Curved section & stations $7-10$ & stations $7-10$ & stations 3-6 & stations $2-6$ \\
\hline Recovery section & stations $11-14$ & stations $12-14$ & stations $7-10$ & stations $7-10$ \\
\hline
\end{tabular}

*Just before the beginning of the curvature. 


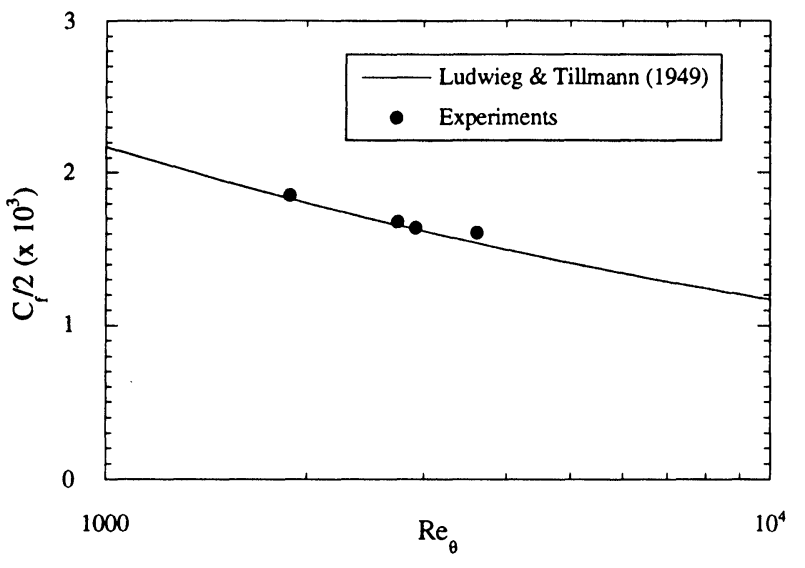

FIGURE 3 Skin friction coefficient vs. momentum thickness Reynolds number-flat developing section.

$$
U / U_{\tau}=U^{+}=f\left(Y^{+}\right)
$$

where

$$
U_{\tau}=\sqrt{\tau_{w} / \rho} \text { and } Y^{+}=\frac{y U_{\tau}}{v}
$$

This is called the law of the wall. The mean velocity distribution in the outer region is expressed as the velocity-defect law:

$$
\frac{U_{e}(x)-U(x, y)}{U_{\tau}}=F(Y)
$$

where $Y=y / \delta(x)$. The viscous sublayer is a special case of equation (3), where the turbulent shear stress is negligible. This is $U^{+}=Y^{+}$, where $Y^{+}<3$.

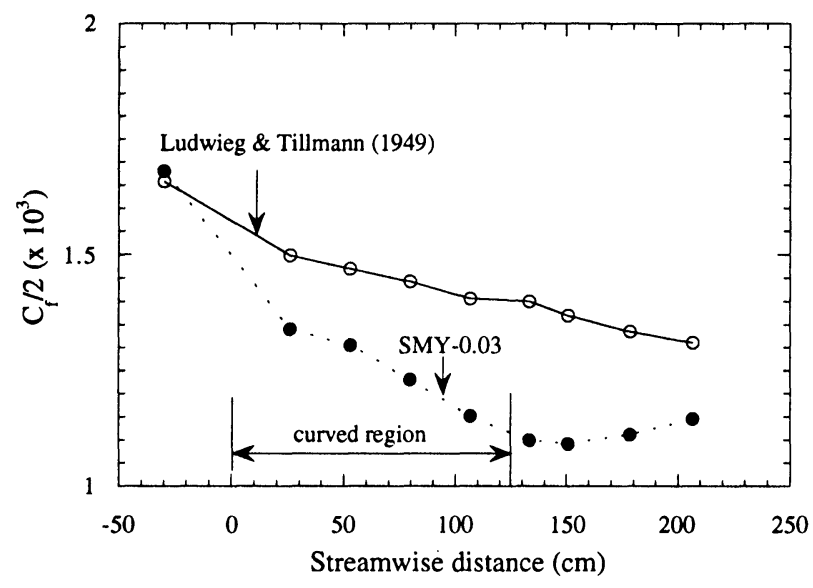

FIGURE 4 Skin friction coefficient variation of SMY-0.03 casedeveloping, curved and recovery sections.
A complete velocity profile requires smooth matching between the inner and outer layers. The inner layer represents the log-linear region and the outer layer, the wake region. The inner variable and the outer variable correlate as

$$
Y=y / \delta=\frac{y U_{\tau} / v}{U_{\tau} \delta / v}=Y^{+} / R e_{\tau}
$$

Now, the inner layer (log-linear region) and the outer layer (wake region) have two distinct scales in a turbulent boundary layer flow. The basic idea of matching between the inner and outer layers is that the outer velocity boundary condition for the inner profile is the same as the inner velocity boundary condition for the outer profile. Based on a singular perturbation method, we can approximate the similarity law of the wake

$$
\frac{U}{U_{\tau}}=(l / \kappa) \ln Y^{+}+C+D
$$

It should be noted the $\kappa$ and $\mathrm{C}$ are the universal constants which are usually taken as 0.41 and 5.0 whereas the constant $\mathrm{D}$ is not universal but dependent on the magnitude of the streamwise pressure gradient, curvature, turbulent intensity etc.

Coles [1956] proposed a mean velocity profile based upon eq. (6):

$$
\frac{U}{U_{\tau}}=(l / \kappa) \ln Y^{+}+C+(\Pi / \kappa) \cdot W(y / \delta)
$$

$\mathrm{W}$ is a function of $y / \delta$ and is found from a fit to experimental data as

$$
W(y / \delta)=1-\cos (\pi y / \delta)
$$

The wake parameter, $\Pi$, can be evaluated at the edge of the boundary layer $(y=\delta)$ using eq. (7). This is:

$$
\frac{U_{e}}{U_{\tau}}=\frac{1}{\kappa} \ln \left(\frac{\delta U_{\tau}}{v}\right)+C+2 \Pi / \kappa
$$

The wake parameter is found by experiment to be about 0.55 for $R e_{\theta}>5,000$ in the flat-plate boundary layer without streamwise pressure gradient and curvature. Coles [1962] provided the wake parameter variation with low $\operatorname{Re}_{\theta}$ values. The variation of the wake parameter with the momentum thickness Reynolds number can be expressed as

$$
\begin{aligned}
\Pi & =0.55 \cdot\{1-\exp (-0.243 \cdot \sqrt{z}-0.298 \cdot z)\}, \text { where } \\
z & =\left(\operatorname{Re}_{\theta} / 425-1\right)
\end{aligned}
$$

So eqs. (7) and (8) with the wake parameter of eq. (10) represents the mean velocity distribution across the 
flat-plate turbulent boundary layer with zero pressure gradient.

\section{Curvature Effect on the Wake: Theory}

Coles [1968] made an extensive survey of many experiments and reported flows in or near equilibrium where the wake parameter is expected to be constant, its value depending on the strength of streamwise pressure gradient. In nonequilibrium boundary layers, $\Pi$ depends on streamwise distance $(x)$. Turbulent flows on the curved wall and following recovery wall should be considered nonequilibrium cases. Therefore, the wake parameter may depend on the strength of curvature, $\delta / R$, which is the function of $x$.

For the stations in the developing and recovery flat walls, the wake parameters are calculated by substituting the measured boundary layer thicknesses, friction velocities, and potential velocities into eq. (9), which can be written as

$$
\Pi=(\kappa / 2)\left[\frac{U_{e}}{U_{\tau}}-\left\{\frac{1}{\kappa} \ln \left(\frac{\delta U_{\tau}}{v}\right)+C\right\}\right]
$$

Whereas, for the stations within the curved region, the same method was used to obtain the wake parameter, assuming the existence of Coles wake law (eq.(8)) in the curved boundary layer. The potential velocity, $U_{p}$ at a location $y$, can be expressed by $U_{p}=[R /(R+y)] \cdot U_{p w}$. Substitution of $U_{e}\left(=U_{p}\right)$ into eq. (11) gives a wake parameter:

$\Pi=(\kappa / 2) \cdot\left[\frac{U_{p w}}{U_{\tau}} \frac{1}{1+\delta / R}-\left\{(1 / \kappa) \cdot \ln \left(\delta U_{\tau} / v\right)+C\right\}\right]$

Then the profiles of wake components are calculated by subtracting out the log-linear part from dimensionless velocity profile, $U / U_{\tau}$ :

$W(y / \delta)=(\kappa / \Pi) \cdot\left[\frac{U}{U_{\tau}}-\left\{(1 / \kappa) \ln Y^{+}+C\right\}\right]$

This experimental wake profiles in the developing, curved and recovery walls are compared to Coles wake law, next.

\section{Law of the Wake for Developing Section: Experiments}

Figure 5 shows the comparison of inverse-wake-parameter, 1/П, between the Coles correlation and presently selected four experimental data in the flat, developing region. For flows over the flat plate with zero pressure gradient, wake parameter, $\Pi$, can be correlated to skin friction coefficient, $C_{f} / 2$, using equations (2) and (10). When the momentum thickness Reynolds number is greater than $5000,1 / \Pi$ is a constant, equal to 1.82 . It is the equilibrium value for the fully turbulent boundary layer suggested by Coles in 1962. Experimental wake values for the developing section calculated from eq. (11) are plotted in the figure against the measured skin friction values. It can be observed that the experimental data agree well with the values from eqs. (2) and (10). One case (TWS-0.05) was somewhat away from the matured turbulent boundary layer, and the other three cases were apparently close to the fully turbulent flowindicating $1 / \Pi$ is close to 1.82 .

Velocity profile of station 1 of SMY-0.01 case is plotted, in Fig. 6, using inner coordinates $\left(U^{+}\right.$vs. $\left.Y^{+}\right)$and, in Fig. 7, the wake components (the rise above the log-linear line) are shown based upon outer coordinates $(Y / \delta)$. This station is located in the developing region. Figure 6 shows that the data follow nicely the turbulent log-linear line for low $Y^{+}$values. In the outer region $\left(Y^{+}\right.$ $>200$ ), however, one sees that the data points are higher than the log-linear line by the amount of wake components. The differences are the wake component profile and it is compared with the Coles wake profile, $W(y / \delta)=$ $1-\cos (\pi y / \delta)$ in Fig. 7. As demonstrated in this figure, the Coles wake profile fits the experimental data well in the flat-plate, developing section. In Fig. 6, notice that the wake component is only $\sim 10 \%$ of the magnitude of the velocity profile. Therefore, the agreement shown in the Fig. 7 is almost perfect in terms of the overall velocity.

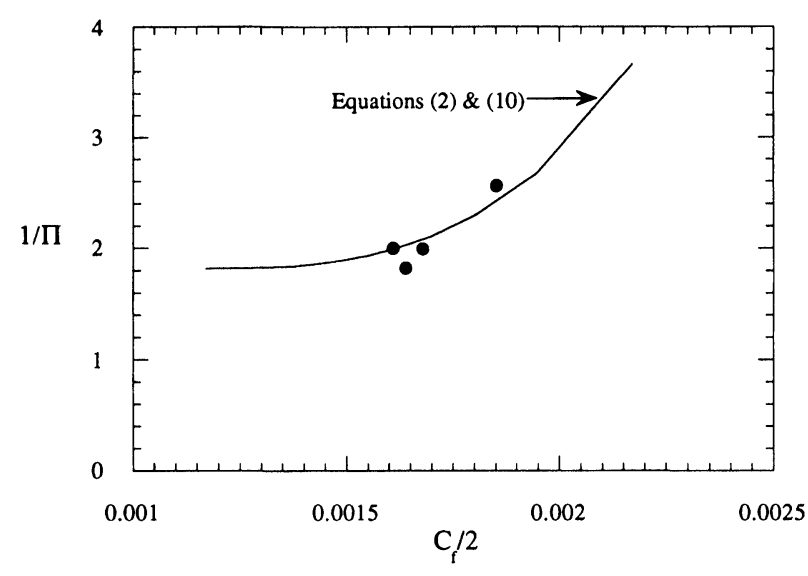

FIGURE 5 Wake parameter vs. skin friction coefficient-flat developing section. 


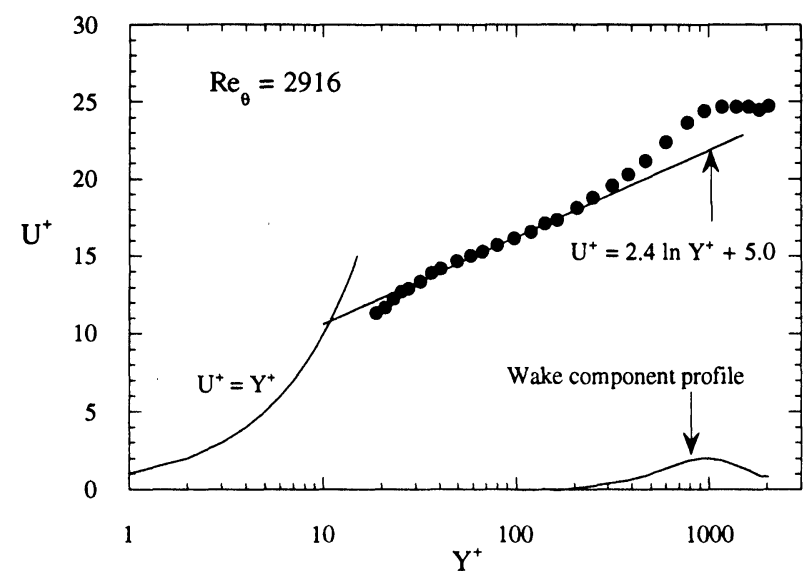

FIGURE 6 Velocity profile and wake component-station 1 (developing region) of SMY-0.01.

\section{Law of the Wake for Curved and Recovery Sections: Experiments}

Figures 8, 9, 10 and 11 indicate that Coles wake law (eq. (8)) is valid in the curved wall boundary layer and, also, in the recovering boundary layer for the range of curvature from 0.01 to 0.078 (SMY-0.01, SMY-0.03 and TWS- 0.05 in Table I). In plotting these wake components, W, eq. (12) was used to determine the experimental wake parameter values. Coles wake law with the varying wake parameter, depending upon the curvature strength, shows an agreement within $6 \%$ of mean velocity when compared with the measured profiles' wake components for the three weaker curvatures (SMY-0.01, SMY-0.03 and TWS-0.05). It appeared to be worse than it actually is because the wake component shows only

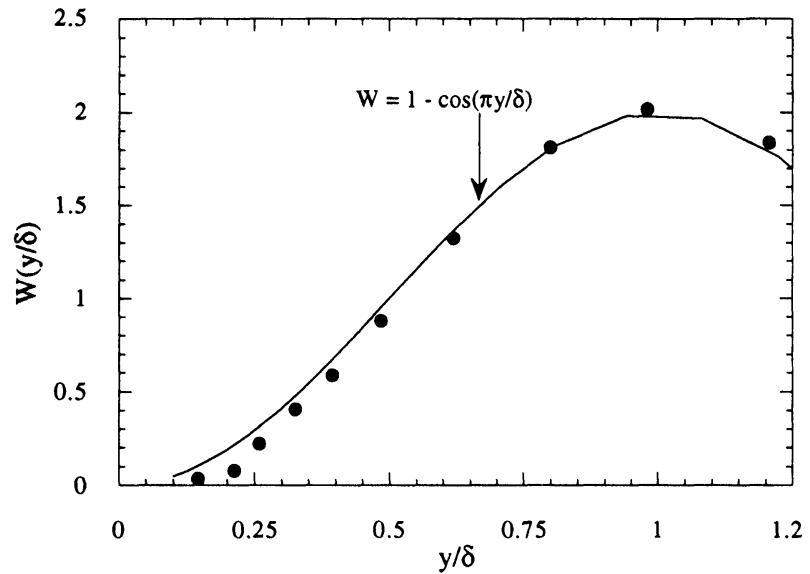

FIGURE 7 The law of the wake-station 1 (developing region) of SMY-0.01.

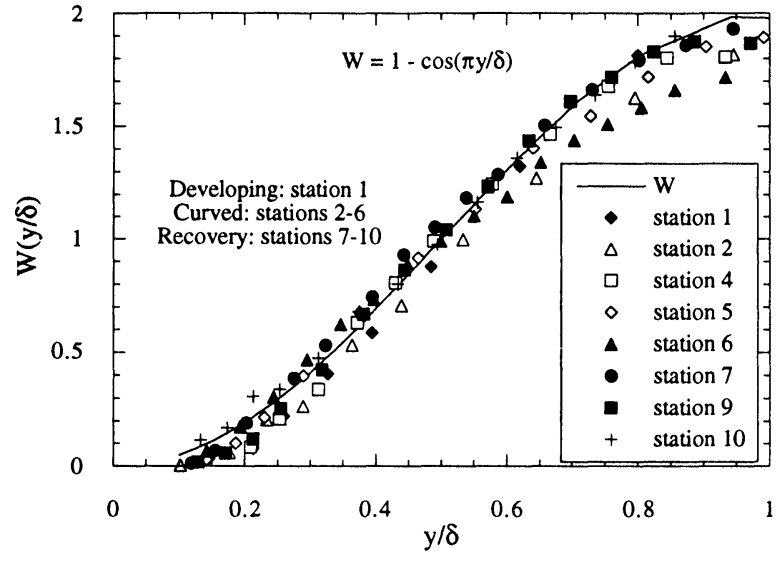

FIGURE 8 Wake component profile-developing, curved and recovery sections-SMY-0.01.

the rise above the log-linear line as stated in the Fig. 6 discussion. The distribution of the wake components in the strongly-curved case (TWS-0.1) apparently does not indicate the similarity with Coles wake law-see Fig. 11.

Given that Coles wake law represents the flows on the curved and recovery walls reasonably well for $\delta / R<$ 0.078 , one can investigate the trends in the wake parameter; particularly the trends due to the streamwise curvature. Figure 12 expresses the variation of $1 / \Pi$ and $C_{f} / 2$ for all measured stations in SMY-0.03 to illustrate predictability in the behavior of wake in the curved and recovery sections. The flat pre-plate data (negative $\mathrm{x}$ location in Fig. 12) is comparable with the equilibrium value, $\Pi=0.55$. As flow enters into the curve, both $1 / \Pi$ and $C_{f} / 2$ indicated monatomic decreases. These two variables, again, showed consistently slow recovery from the effects of convex curvature. The same trends were

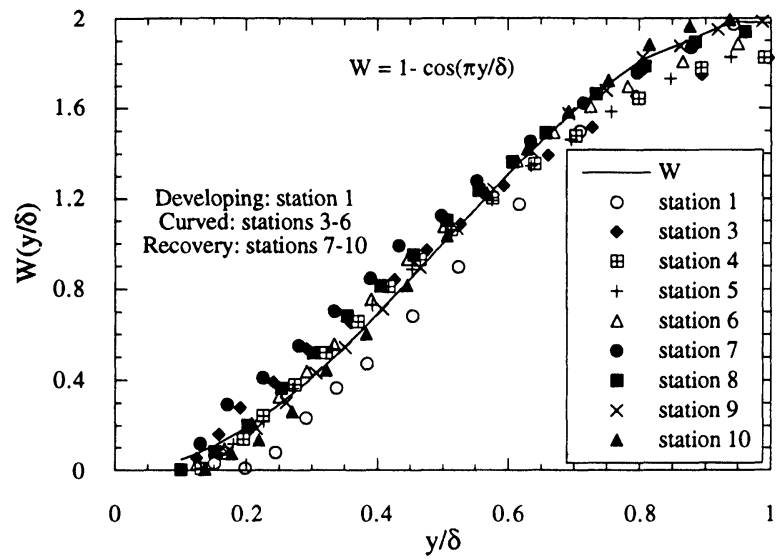

FIGURE 9 Wake component profile-developing, curved and recovery sections-SMY-0.03. 


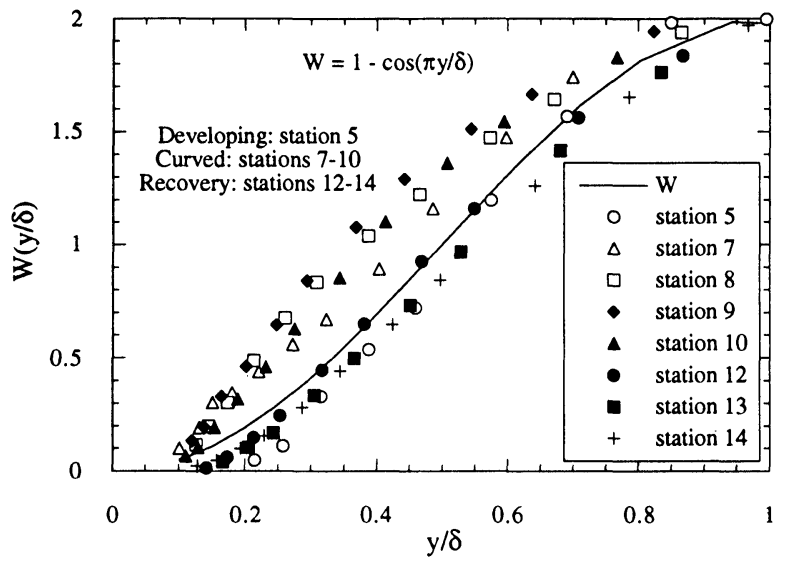

FIGURE 10 Wake component profile-developing, curved and recovery sections-TWS- 0.05 .

observable for the three weaker curvatures. Since Coles wake law is valid, the variations of $1 / \Pi$ and $C_{f} / 2$ depend only upon $R e_{x}$ for both curved and recovery sections. The same trends were seen when these variables were plotted against momentum thickness Renolds number.

The next step is correlating skin friction coefficient with wake parameter for the cases of strength of curvature less than 0.078. A relationship is sought to describe the deviation from the flat-plate equilibrium turbulent boundary layer. All stations available within curved and recovery sections in the three experiments (SMY-0.01, SMY-0.03 and TWS-0.05), are employed to compute the dimensionless wake parameter variation versus dimensionless skin friction coefficient shown in Fig. 13. Characteristic skin friction coefficients and inverse

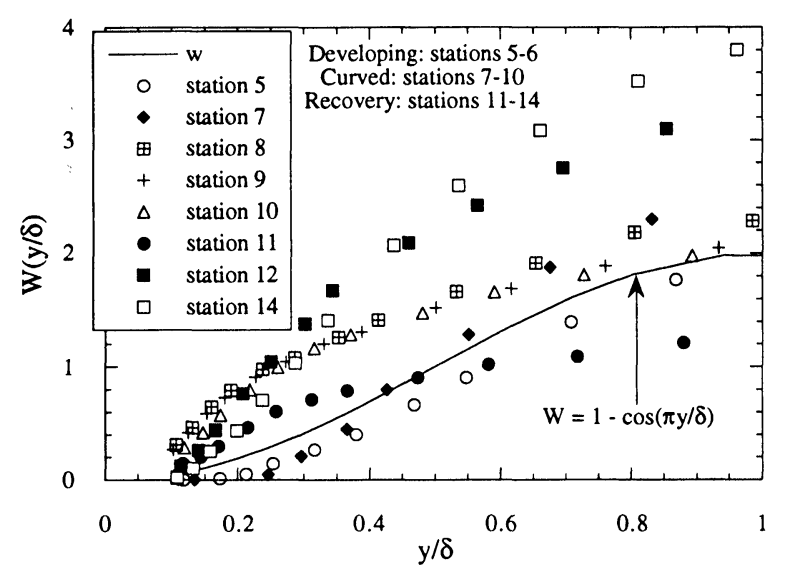

FIGURE 11 Wake component profile-developing, curved and recovery sections-TWS- 0.1

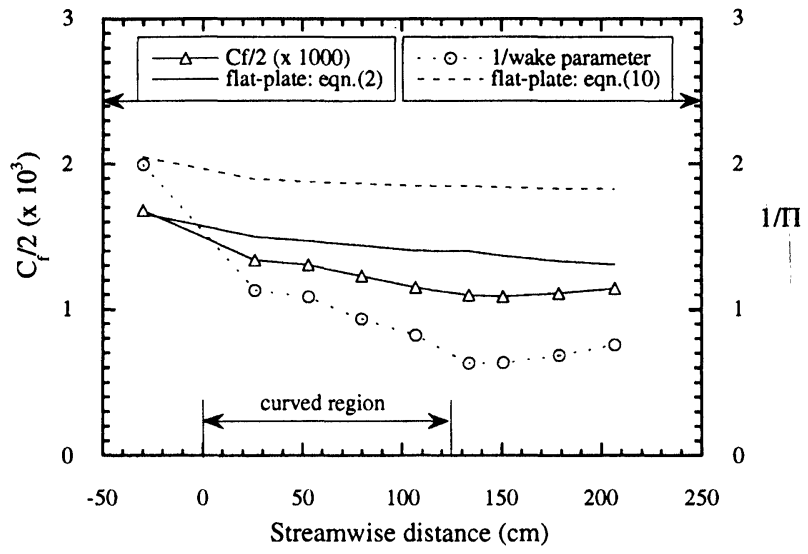

FIGURE 12 The curvature effect on the wake and skin friction coefficient-SMY-0.03.

wake parameter values used for nondimensionalization were determined from the flat-plate correlation in eqs. (2) and (10), respectively. As indicated clearly in Fig. 13, inverse wake parameter is correlated with skin friction coefficient by:

$$
(1 / \Pi) /(1 / \Pi)_{\text {flat }}=2.27 C_{f} /\left(C_{f}\right)_{\text {flat }}-1.09
$$

In the figure, one can see the degree of influence due to curvature in the curved and recovery sections by simply reading the coordinates. The y-axis, $(1 / \Pi) /(1 / \Pi)_{f a t}$, indicates the degree of influence on the wake (outer layer) whereas the x-axis, $C_{f} /\left(C_{f}\right)_{f a t}$, indicates that on the wall shear stress (inner layer). These two dimensionless variables are found to be linearly proportional.

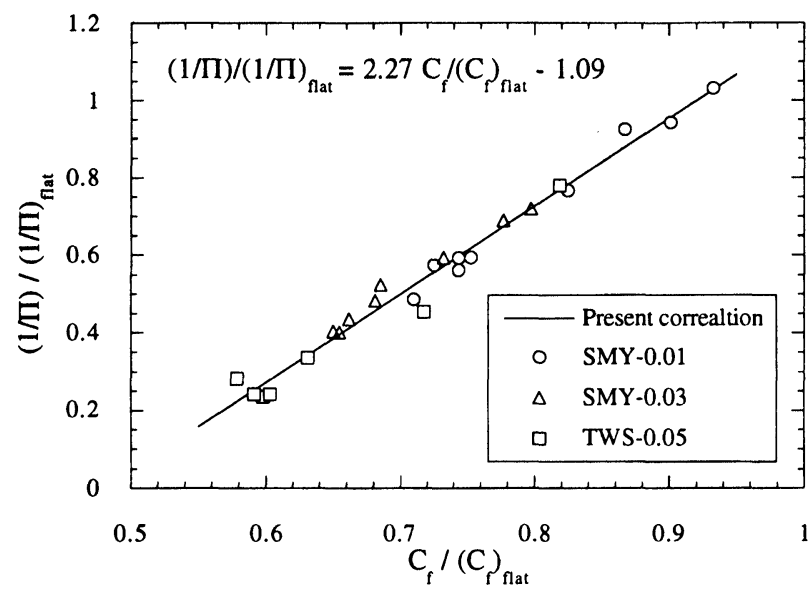

FIGURE 13 Wake parameters vs. skin friction coefficient for 0.01 $<y / d<0.078$-SMY-0.01, SMY-0.03, and TWS-0.05. 
It should be pointed out in Fig. 14 that the present correlation for the dimensionless inverse wake parameter and skin friction coefficient is not satisfactory for the strongly curved case, TWS-0.1. This was expected from the validity check of Coles wake law in Fig. 10. Especially, the experimental values at station 7 shown in Fig. 14 was found further away from the proposed relationship unlike the other stations. Station 7 is $10 \mathrm{~cm}$ downstream of the entrance to the strong convex curve $(\delta / R=0.1)$. This observation coincides with the shear stress profiles by Gillis and Johnston [1983]. For the strong case $(\delta / R=0.1)$, they have reported that the shear stress reverses sign within the curved section unlikely those of the weaker strengths of curvatures. This may indicate that a distinction should be made when the curvature strength approaches 0.1 .

The present observation, for $0.01<\delta / R<0.078$, implies that curvature effects on the turbulent boundary layer flow upon entering and also leaving the convex curve can be represented by a simple linear relationship between the inner and outer layers. According to the turbulence intensity $\left(u^{\prime}\right)$ profiles in Fig. 15 by You et al. [1986], variations in the shape of the profiles were divided into 4 steps:

1. Upon entering the curve, a near wall peak dramatically drops while the remainder of the profile $(y / \delta>0.1)$ is hardly affected.

2. The later portion of the curved section indicates complete response of the outer region in the $u^{\prime}$ profiles, showing a self-similar shape of the turbulence intensity profiles.

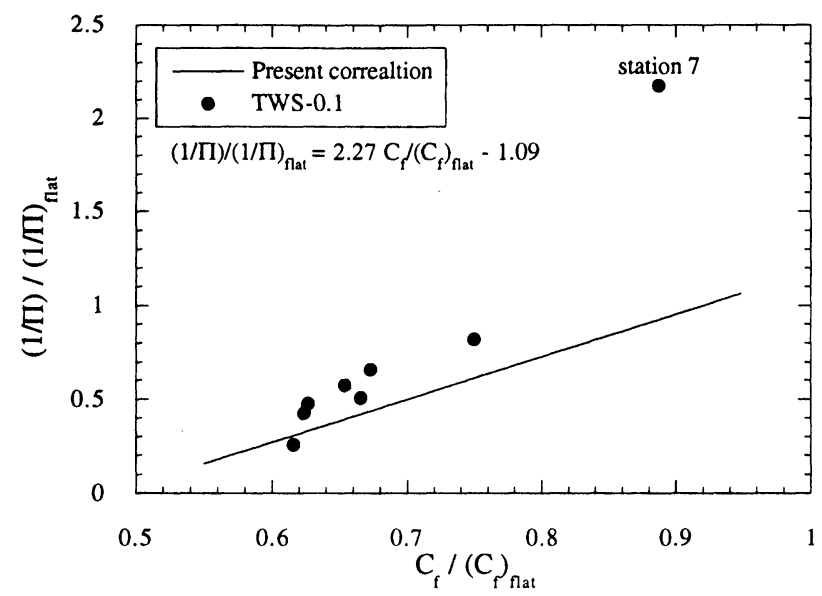

FIGURE 14 Wake parameters vs. skin friction coefficient for 0.071 $<y / d<0.113$-TWS-0.1.
3. In returning to flat-plate recovery, a peak appears at $y / \delta=0.2$ at the beginning of the recovery.

4. The peak diffuses slowly outward.

It is interesting to note that, by the end of the test section, the recovery of turbulence intensity is complete beyond $y / \delta=0.2$, but the near-wall data show no change from profiles taken at the beginning of recovery. You et al. [1986] also confirmed the similar interactions between inner and outer layers by the normal component velocity fluctuation and shear stress profiles, consistently characterized by the quick reduction near the wall, followed by gradual adjustment in the outer layer, and slow recovery. In spite of the complex flow upon entering and leaving the curve, the present correlation (eq. (14)) suggests a simple global relationship between the inner and outer layers.

\section{CONCLUSIONS}

The present study is an analysis of the nature of turbulent boundary layers on convexly curved and flat-recovery surfaces. Especially, it is to investigate flow characteristics at the recovery section upon withdrawal of convex curvature where the understanding is poor. An effort is made to correlate the wake and wall functions in both curved and recovery regions of turbulent boundary layers. Important findings are:

- Coles wake law, $W(y / \delta)=1-\cos (\pi y / \delta)$, can represent the flows on the curved and recovery walls for the strength of curvature ranging $0.01 \sim 0.078$. Wake parameters are evaluated by:

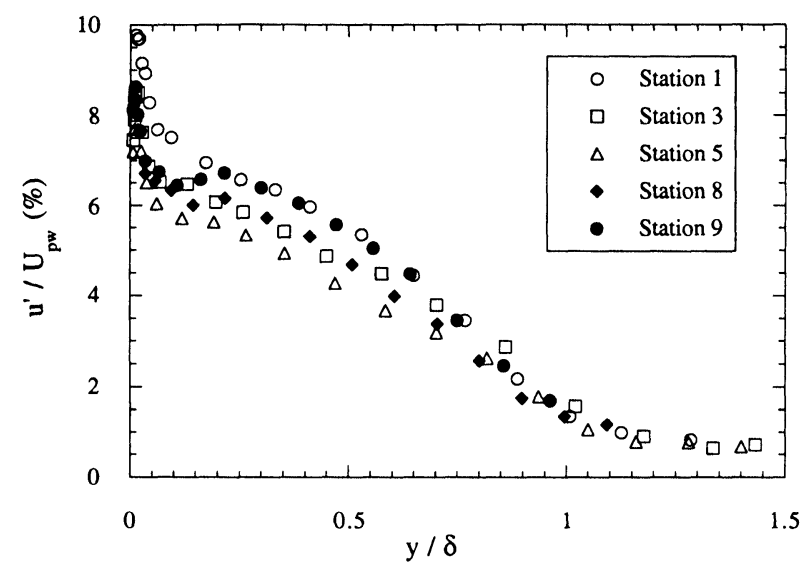

FIGURE 15 Streamwise-normal turbulence intensity profiles-SMY0.03 . 


$$
\Pi=(\kappa / 2) \cdot\left[\frac{U_{p w}}{U_{\tau}} \frac{1}{1+\delta / R}-\left\{(1 / \kappa) \cdot \ln \left(\delta U_{\tau} / v\right)+C\right\}\right]
$$

At higher strengths of curvature, approaching 0.1 , it fails.

- Dimensionless inverse wake parameter is found to be proportional to dimensionless skin friction coefficient for the flows in curved and recovery sections by:

$$
(1 / \Pi) /(1 / \Pi)_{\text {flat }}=2.27 C_{f} /\left(C_{f}\right)_{\text {flat }}-1.09
$$

This relationship is applicable for the flows on the curved and recovery walls for $\delta / R<0.078$.

- In the present observation for $0.01<\delta / R<0.078$, it is interesting to note that, in spite of the complex interactions between inner and outer layers by the normal component velocity fluctuation and shear stress profiles, the present correlation suggests a simple global correlation between the inner and outer layer, especially, in the recovery process from the convex curvature.

$\begin{array}{ll}\text { Nomenclature } & \\ C_{f} / 2 & \text { Skin friction coefficient } \\ H & \text { Shape factor } \\ l & \text { Mixing length } \\ l_{o} & \text { Flat-wall mixing length } \\ R & \text { Wall radius of curvature } \\ R e & \text { Reynolds number } \\ R i & \text { Richardson number, eq. (1) } \\ U & \text { Streamwise mean velocity } \\ U^{+} & \text {Normalized streamwise velocity, } U / U_{\tau} \\ U_{\tau} & \text { Shear velocity, } \sqrt{\left(\tau_{u^{\prime}} / \rho\right)} \\ u^{\prime} & \text { Root-mean-square of fluctuating streamwise } \\ & \text { velocity } \\ -u^{\prime} v^{\prime} & \text { Reynolds shear stress } \\ W & \text { Wake component } \\ x & \text { Streamwise distance } \\ y & \text { Distance normal to the test wall } \\ Y & y / \delta \\ Y^{+} & \text {Normalized distance, } y U_{\tau} / \nu\end{array}$

\section{Greek Letters}

$\beta$
$\delta$
$\theta$
$\kappa$
$\Pi$
$\nu$
$\rho$
$\tau$

\author{
Subscripts \\ fla \\ $e, p$
}

$w$

Flat-wall

Potential flow

Wall value

\section{References}

Adams, E. W. and Johnston, J. P., 1981. A Mixing-Length Model for the Prediction of Convex Curvature Effects on Turbulent Boundary Layers, Rept IL-42, Stanford University.

Bradshaw, P., 1969. The Analogy between Streamwise Curvature and Buoyancy in Turbulent Shear Flow, J. Fluid Mechanics, Vol. 36, part 1, pp. 177-191.

Bradshaw, P., 1972. Effects of streamline Curvature on Turbulent Flow, AGARDograph No. 169.

Coles, D., 1956. The Law of the Wake in the Turbulent Boundary Layer, J. Fluid Mechanics, Vol. 1, pp. 191-226.

Coles, D., 1962. The Turbulent Boundary Layer in a Incompressible Fluid, Rand Corp., Rept. R-403-PR.

Coles, D., 1968. Computations of Turbulent Boundary Layers, Proceedings of the Stanford Conference, AFOSR-IFP, Vol. II.

Ellis, L. B. and Joubert, P. N., 1974. Turbulent Shear Flows in a Curved

Duct, J. Fluid Mechanics, Vol. 62, part 1, pp. 54-84.

Gillis, J. C. and Johnston, J. P., 1981. Turbulent Boundary Layer on a Convex, Curved Surface, NASA-CR3391, March.

Gillis, J. C. and Johnston, J. P., 1983. Turbulent Boundary-Layer Flow and Structure on a Convex Wall and its Redevelopment on a Flat Wall, J. Fluid Mechanics, Vol. 135, pp. 123-153.

Hoffmann, P. H. and Bradshaw, P., 1978. Turbulent Boundary Layers on Surfaces of Mild Longitudinal Curvature, Imperial College, Aero. Rept. 78-04, Dec.

Kim, J. and Simon, T. W., 1987. Measurements of Turbulent Transport of Heat and Momentum in Convexly Curved Boundary Layers: Effects of Curvature, Recovery and Free-stream Turbulence, ASME paper 87-GT-199.

Launder, B. E., Priddin, C. H. and Sharma, B. I., 1977. The Calculation of Turbulent Boundary Layer on Spinning and Curved Surfaces, J. Fluid Engineering, Vol. 99, p. 231.

Ludwieg, H. and Tillmann, W., 1950. Untersuchungen uber die Wandsschubspannung in Turbulenten Reibungsschichten, Ing. Arch. 17, pp. 288-299 (1949)-English translation in NACA TM 1285.

Muck, K. C., 1982. Turbulent Boundary Layers on Mildly Curved Surfaces, Ph.D. Thesis, Dept. of Aeronautics, Imperial College.

Rodi, W. and Scheuerer, G., 1983. Calculation of Curved Shear Layers with Two-Equation Turbulence Models, Phys. Fluids, Vol. 26, p. 1422.

Schlichting, H., 1951. Boundary Layer Theory, 7th ed., McGraw Hill, p. 525 .

Schmidbauer, H., 1936. Behavior of Turbulent Boundary Layers on Curved Convex Walls, NACA-TM No. 791.

Simon, T. W. and Moffat, R. J., 1982a. Convex Curvature Effects on the Heated Turbulent Boundary Layers, Heat Transfer 1982, Vol. 3, pp. 295-301, Hemisphere Publishing Corp.

Simon, T. W., Moffat, R. J., Johnston, J. P. and Kays, W. M., 1982b. Turbulent Boundary Layer Heat Transfer Experiments: Convex Curvature Effects Including Introduction and Recovery, NASA CR3510, Feb.

So, R. M. C. and Mellor, G. L., 1973. Experiment on Convex Curvature Effects in Turbulent Boundary Layers, J. Fluid Mechanics, Vol. 60, part 1, pp. 43-62.

Thomann, H., 1968. Effect of Streamwise Wall Curvature on Heat Transfer in a Turbulent Boundary Layer, J. Fluid Mechanics, Vol. 33, pp. 283-292. 
Wendt, F., 1933. Turbulente Stromungen Zwischen Zesi Rotierender Honaxialen Zylindren, Ing-Arch., Vol. 4, p. 577.

Wilcken, H., 1967. Turbulente Grenzschichten an Gewölbten Flächen Berlin, Vol. 1, No. 4, Sept. (1930)-translated as NACA TT-F-11, 421 .
You, S. M., Simon, T. W., and Kim, J., 1986. Boundary Layer Heat Transfer and Fluid Mechanics Measurements on a Mildly-Curved Convex Wall, Heat Transfer 1986, Vol. 3, pp. 1089-1094, Hemisphere Publishing Corp. 

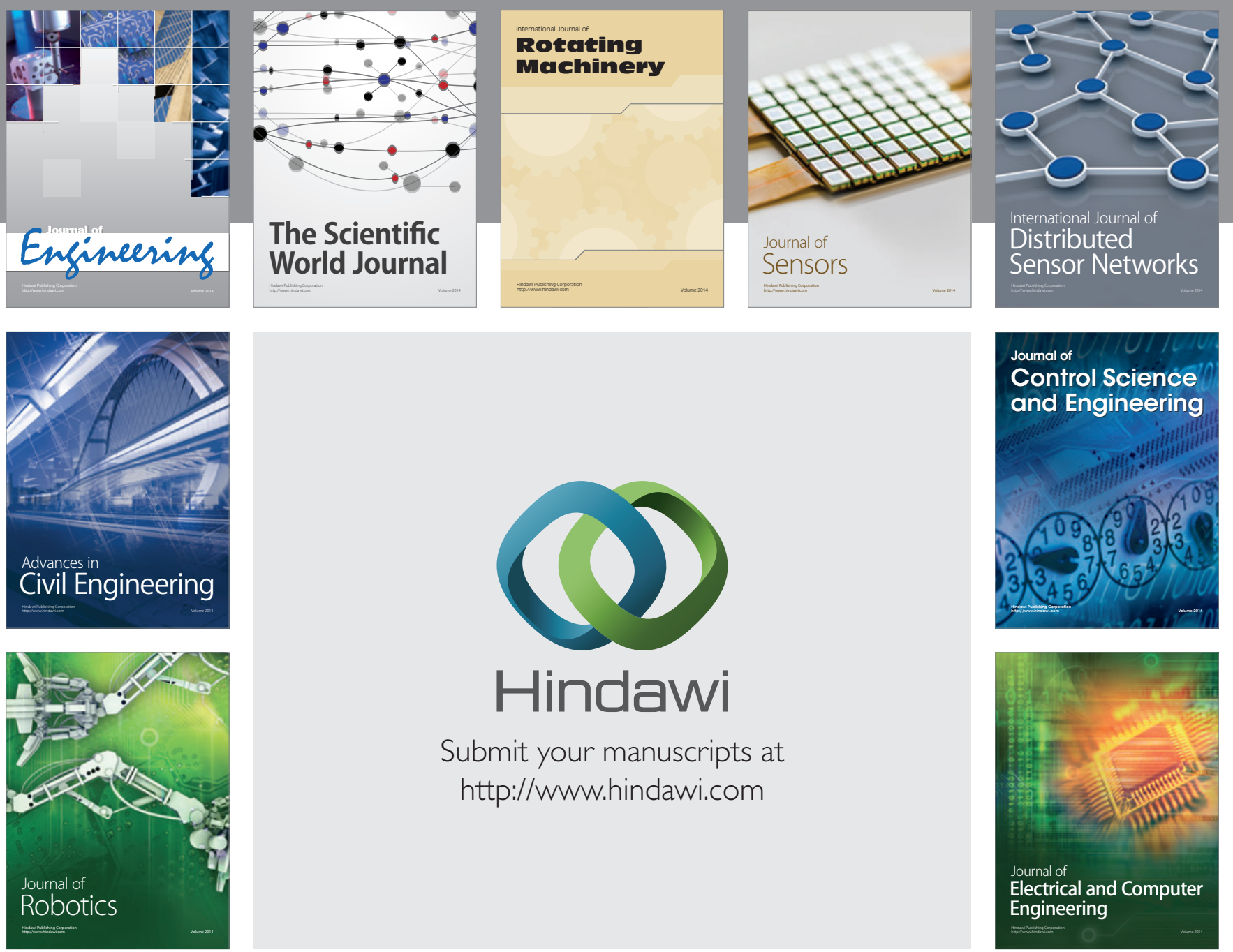

Submit your manuscripts at

http://www.hindawi.com
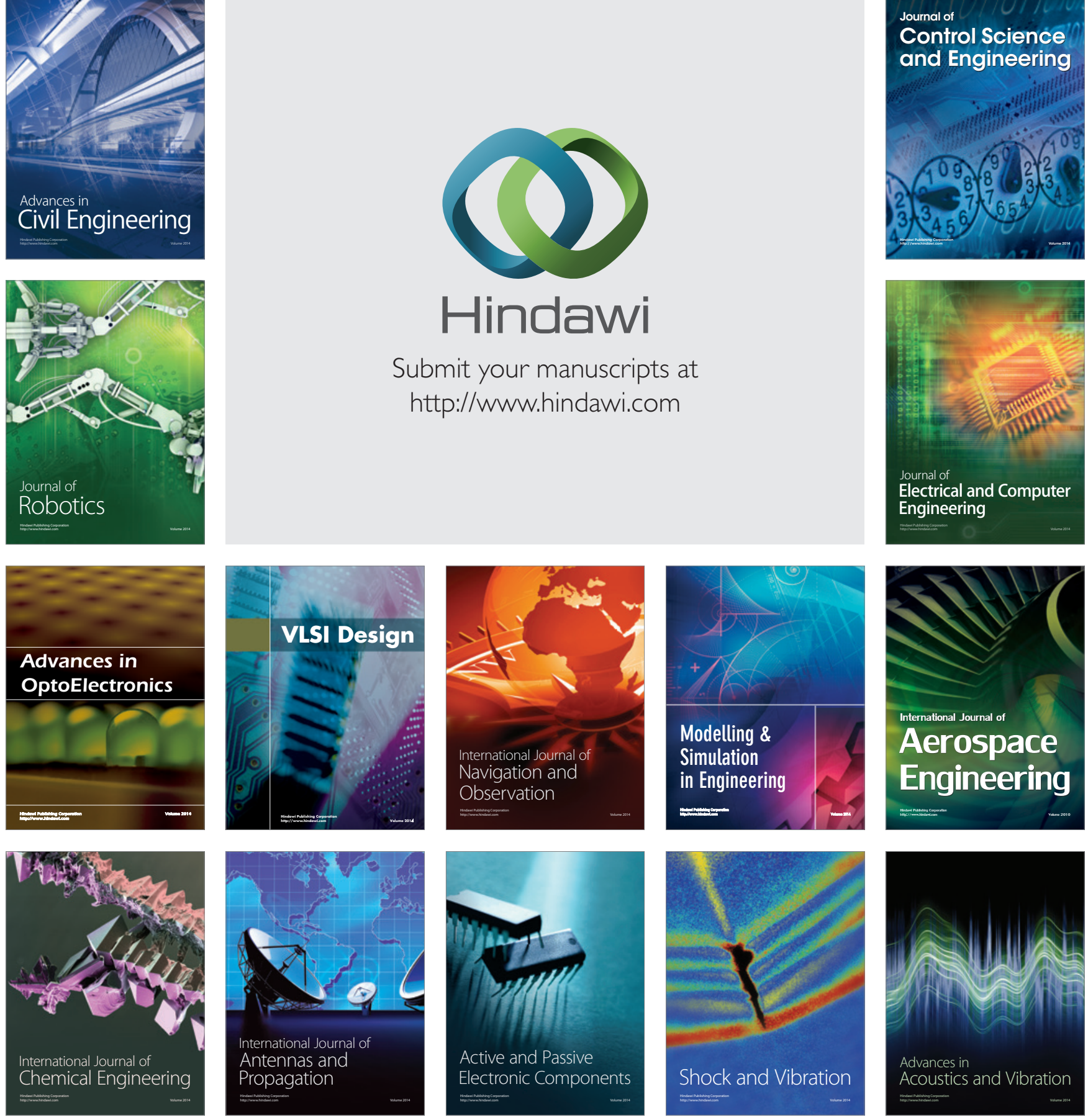